\title{
Activated fibronectin-secretory phenotype of mesenchymal stromal cells in pre-fibrotic myeloproliferative neoplasms
}

\author{
Rebekka K Schneider ${ }^{1,2^{*}+}$, Susanne Ziegler ${ }^{3+}$, Isabelle Leisten ${ }^{2}$, Monica SV Ferreira ${ }^{2,3}$, Anne Schumacher ${ }^{3}$, \\ Björn Rath ${ }^{4}$, Dirk Fahrenkamp ${ }^{5}$, Gerhard Müller-Newen ${ }^{5}$, Martina Crysandt ${ }^{3}$, Stefan Wilop ${ }^{3}$, Edgar Jost ${ }^{3}$, \\ Steffen Koschmieder ${ }^{3}$, Ruth Knüchel ${ }^{2}$, Tim H Brümmendorf ${ }^{3}$ and Patrick Ziegler ${ }^{3}$
}

\begin{abstract}
We characterized bone marrow stromal cells (BMSC) from patients with pre-fibrotic myeloproliferative neoplasms (MPN). MPN-BMSC showed decreased capacity to stimulate the proliferation of colony-forming units of normal hematopoietic stem and progenitor cells and displayed increased matrix remodelling (in particular fibronectin deposition) compared to control BMSC. This finding was confirmed in pre-fibrotic MPN bone marrow biopsies in a tissue microarray $(n=34)$, which stained positive for fibronectin in the absence of reticulin as a standard myelofibrosis marker. Fibronectin expression correlated significantly with reduced haemoglobin levels in MPN-patients $(p=0.007 ; R 2=0.42)$. Our data show significant cell-intrinsic alterations in MPN-MSC and suggest that Fibronectin expression might be applicable as a biomarker for the identification of early myelofibrotic transformation in reticulin-negative MPN.
\end{abstract}

Keywords: Myeloproliferative neoplasms, Myelofibrosis, Mesenchymal stromal cells, Tissue microarray biomarker, Fibronectin

\section{Findings}

It remains elusive if human bone-marrow mesenchymal stromal cells (BMSC) display an intrinsic role in the development of myelofibrosis independent of the malignant clone. We applied a three-dimensional collagen culture-system as an in vitro-screening for the functional characterization of BMSC isolated from MPN-patients $[1,2]$. The assay was designed to be applicable for potential diagnostic and prognostic utility in the investigation of progressive myelofibrosis. The main question of the study was if MPN-MSCs functionally differ from MSCs isolated from healthy donors in (i) their matrix remodelling and (ii) hematopoiesis-supporting capacity. MSC were isolated from patients with ET $(\mathrm{n}=4), \operatorname{PV}(\mathrm{n}=5)$, CML $(n=5)$, all without or only mild myelofibrosis, one

\footnotetext{
* Correspondence: reschneider@ukaachen.de

${ }^{\dagger}$ Equal contributors

'Division of Hematology, Department of Medicine, Brigham and Women's Hospital, Harvard Medical School, 1 Blackfan Circle, Boston, MA 02115, USA ${ }^{2}$ Institute of Pathology, University Hospital Aachen, RWTH Aachen University, Pauwelsstrasse 30, Aachen 52074, Germany

Full list of author information is available at the end of the article
}

patient with post essential thrombocythemia myelofibrosis (ET-MF, $\mathrm{n}=1$ ) as well as from control patients (nonMPN, $n=6$ ), (Figure 1a). MSC were isolated from bone marrows by plastic adherence. No significant differences were detected in (1) the number of proliferating mesenchymal precursors (CFU-F), (2) MSC morphology, (3) osteoblastic and adipogenic differentiation or (4) the surface marker pattern of MPN- and control-MSC. In line with previous reports, (RT)-PCR examination of cultured BMSC neither detected the JAK2-V617F mutation nor the BCR-ABL fusion transcript, despite the fact that $3 / 5$ of ET cases and 5/5 of PV were positive for the JAK2-V617F mutation and all CML cases (5/5) were positive for BCR$\mathrm{ABL}$ translocation in peripheral blood cells (Figure 1b).

To characterize the hematopoiesis-supporting capacity of MSC, secreted cytokines and their biological activity were tested. ET-MSC secreted significantly lower levels of G-CSF and IL-7 compared to controls, indicating a defect in the hematopoiesis-supporting capacity (Figure 1c). We therefore evaluated the biological activity of secreted cytokines in myeloid colony-forming unit (CFU) assays 


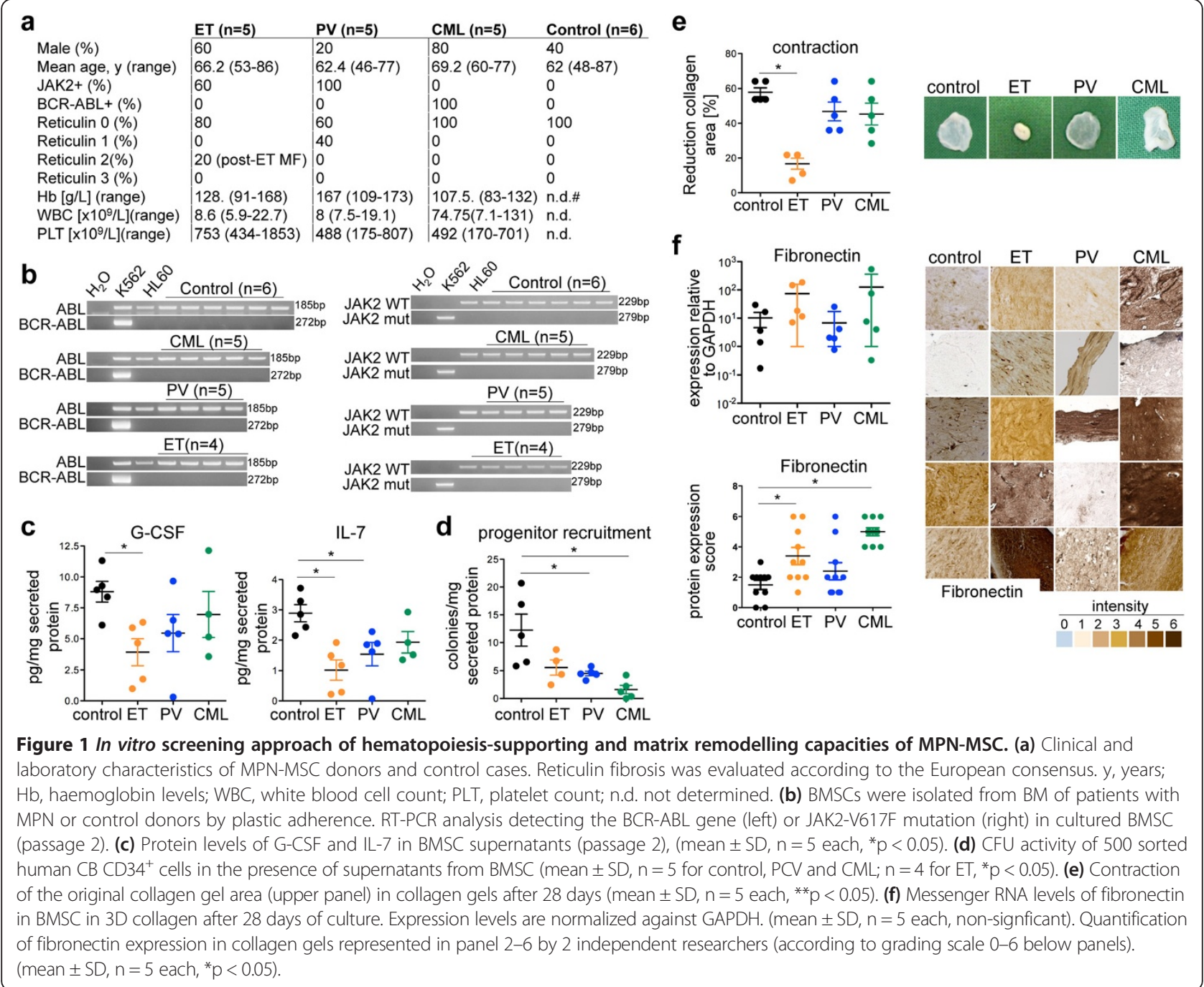

using human cord blood (CB) CD34 ${ }^{+}$-hematopoietic stem and progenitor cells (HSPCs) [3]. Myeloid CFU-activity significantly decreased when supernatants from PV and CML were used (Figure 1d). These findings indicate that MPN-BMSCs lose their capacity to produce and release functional myeloid differentiation-supporting cytokines.

Further, we tested MPN-BMSCs for their capacity to remodel extracellular matrix (ECM) in the absence of the hematopoietic clone [1,2]. MSC isolated from ET induced a significant reduction of the collagen area accompanied by enhanced matrix stiffening (Figure 1e). We analysed if the contraction potential involves matrix production and scored protein and gene expression of matrix components. A significant up-regulation of the ECM protein fibronectin was detected in ET-MSC by immunohistochemistry (Figure 1f). We next asked if ECM remodelling and the significant differential fibronectin expression can be attributed to MSC in correlating bone marrow biopsies of MSC donors. We applied CD271 for the identification of MSC in bone marrow biopsies as this marker most faithfully identifies the heterogenous stromal cell (MSC) population in the human bone marrow up-to-date [4]. Our first finding was that the distribution of $\mathrm{CD} 271^{+}$MSC significantly differed in MPN biopsies compared to the control bone marrow (Figure 2a). In controls, CD271 was mainly expressed in the endosteal and vascular niche [5]. In MPN entities however, CD271 ${ }^{+}$cells were mobilized from these niches and identified in association to dysplastic megakaryocytes (Figure 2a). Our second finding was that fibronectin is expressed in association to CD271 in the hematopoietic niches of the bone marrow in control donors. In striking contrast, fibronectin was diffusely up-regulated in association to expanded stromal cells and identified unbound to the ECM in MPN (Figure 2b) as well as in association to megakaryocytes (Figure 2f).

To validate these findings, we systematically analyzed CD271 and fibronectin in tissue microarrays including ET $(\mathrm{n}=13), \mathrm{PV}(\mathrm{n}=11), \mathrm{CML}(\mathrm{n}=14), \operatorname{PMF}(\mathrm{n}=11)$ and non- 


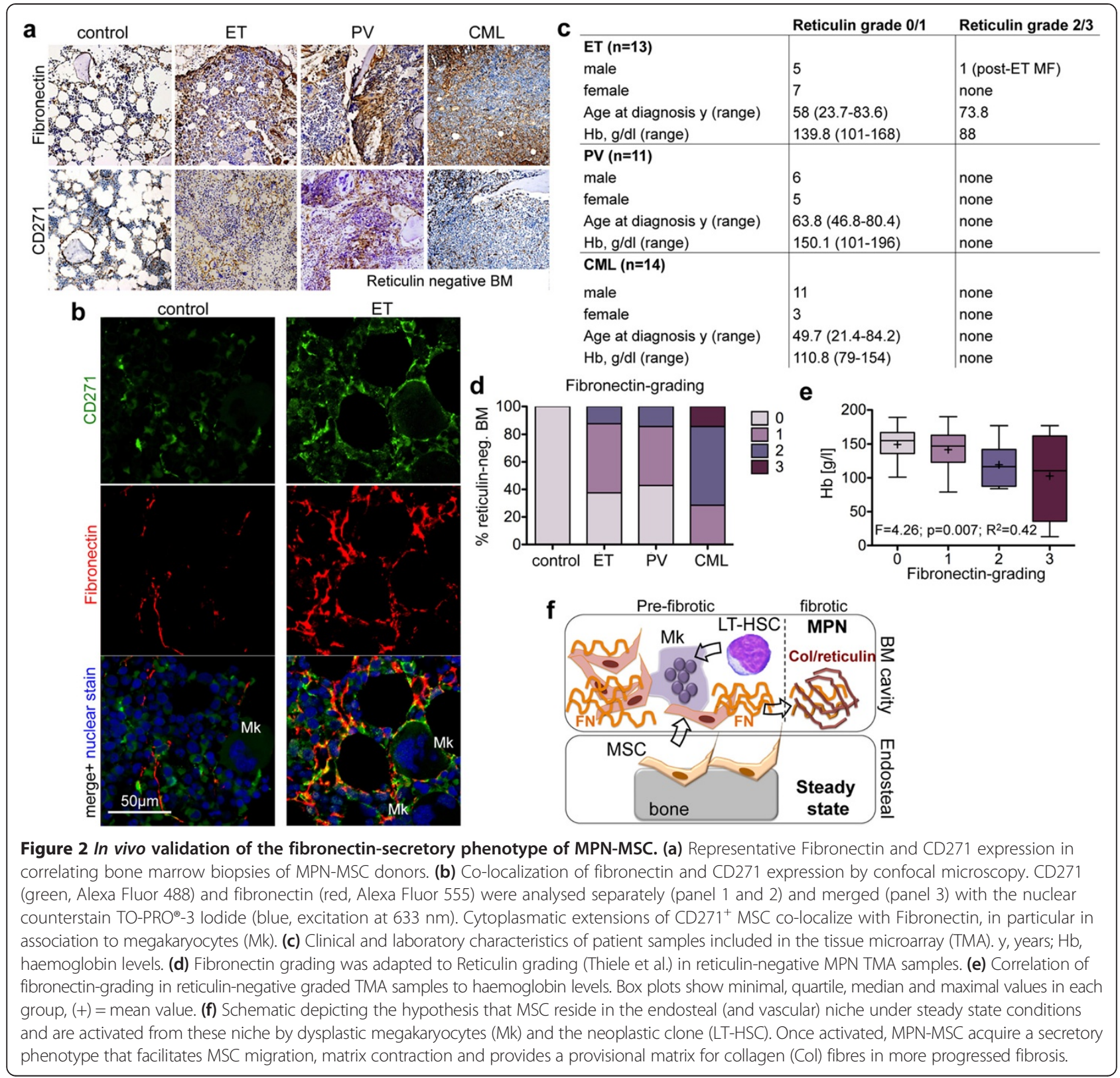

MPN controls ( $\mathrm{n}=17)$, (Figure 2c). CD271 and fibronectin were evaluated applying a grading scheme similar to the established guidelines for grading myelofibrosis $-0=$ no fibrosis and $3=$ severe myelofibrosis [6]. Although the majority of MPN cases were classified as myelofibrosis grade $0-1$ in reticulin-staining, myelofibrotic transformation was graded as $\geq 1$ by Fibronectin-staining in 69\% of ET (vs. 7.7\% Reticulin-representing one post ET-MF patient), 63\% of PV (vs. 0\% Reticulin) and 100\% CML (vs. 0\% Reticulin) cases and by CD271 staining in 54\% of ET, 51\% PV and 64\% CML cases (Figure 2d). In line with the observation of a pre-fibrotic marrow, fibronectin and CD271 expression grade correlated significantly with decreased haemoglobin levels in reticulin-negative biopsies (Figure 2e).
In conclusion, our data reveal an intrinsic defect of MSC in pre-fibrotic MPN resulting in decreased hematopoiesissupporting capacities and increased ECM remodelling. Our data suggest that fibronectin up-regulation/distribution detects early myelofibrotic changes in the BM of MPN patients and implies clinical and prognostic application in different myeloproliferative $\left(\mathrm{Ph}^{-}\right.$and $\left.\mathrm{Ph}^{+}\right)$neoplasms.

\section{Competing interests}

The authors declare that they have no competing interests.

\section{Authors' contributions}

RKS, SZ, THB and PZ designed experiments. RKS, SZ, IL, AS, MSVF, DF, GM-N performed experiments and analyzed data. BR, MC, SW, EJ, SK and RK collected patient samples and clinical information and analyzed these data. RKS and PZ wrote the manuscript. All authors provided critical review of the manuscript. 


\section{Acknowledgments}

This work was supported by the START-program (to RKS and PZ) and the Interdisciplinary Center for Clinical Research (IZKF grant O1-6) both within the Faculty of Medicine RWTH Aachen University. R.K.S was supported by the German Research Foundation (DFG1188/3-1). We thank the "Immunohistochemistry and confocal microscopy facility", a core facility of the Interdisciplinary Center for Clinical Research (IZKF) Aachen within the Faculty of Medicine at RWTH Aachen University. The institutional review board of the medical faculty of the RWTH Aachen approved the project (EK173/06).

\section{Author details}

'Division of Hematology, Department of Medicine, Brigham and Women's Hospital, Harvard Medical School, 1 Blackfan Circle, Boston, MA 02115, USA. ${ }^{2}$ Institute of Pathology, University Hospital Aachen, RWTH Aachen University, Pauwelsstrasse 30, Aachen 52074, Germany. ${ }^{3}$ Department of Oncology, Hematology and Stem cell transplantation, University Hospital Aachen, RWTH Aachen University, Pauwelsstrasse 30, Aachen 52074, Germany. ${ }^{4}$ Department of Orthopaedic Surgery, University Hospital Aachen, RWTH Aachen University, Pauwelsstrasse 30, Aachen 52074, Germany. ${ }^{5}$ Department of Biochemistry and Molecular Biology, University Hospital Aachen, RWTH Aachen University, Pauwelsstrasse 30, Aachen 52074, Germany.

Received: 13 November 2014 Accepted: 29 November 2014

Published online: 14 December 2014

\section{References}

1. Kramann R, Couson SK, Neuss S, Kunter U, Bovi M, Bornemann J, Knuchel R, Jahnen-Dechent W, Floege J, Schneider RK: Exposure to uremic serum induces a procalcific phenotype in human mesenchymal stem cells. Arterioscler Thromb Vasc Biol 2011, 31(9):e45-54.

2. Schneider RK, Puellen A, Kramann R, Raupach K, Bornemann J, Knuechel R, Perez-Bouza A, Neuss S: The osteogenic differentiation of adult bone marrow and perinatal umbilical mesenchymal stem cells and matrix remodelling in three-dimensional collagen scaffolds. Biomaterials 2010, 31(3):467-480

3. Manz MG, Miyamoto T, Akashi K, Weissman IL: Prospective isolation of human clonogenic common myeloid progenitors. Proc Natl Acad Sci U S A 2002, 99(18):11872-11877.

4. Tormin A, Li O, Brune JC, Walsh S, Schutz B, Ehinger M, Ditzel N, Kassem M, Scheding S: CD146 expression on primary nonhematopoietic bone marrow stem cells is correlated with in situ localization. Blood 2011, 117(19):5067-5077.

5. Lo Celso C, Fleming HE, Wu JW, Zhao CX, Miake-Lye S, Fujisaki J, Cote D, Rowe DW, Lin CP, Scadden DT: Live-animal tracking of individual haematopoietic stem/progenitor cells in their niche. Nature 2009 457(7225):92-96

6. Thiele J, Kvasnicka HM, Facchetti F, Franco V, van der Walt J, Orazi A European consensus on grading bone marrow fibrosis and assessment of cellularity. Haematologica 2005, 90(8):1128-1132.

doi:10.1186/s13045-014-0092-2

Cite this article as: Schneider et al:: Activated fibronectin-secretory phenotype of mesenchymal stromal cells in pre-fibrotic myeloproliferative neoplasms. Journal of Hematology \& Oncology 2014 7:92

\section{Submit your next manuscript to BioMed Central and take full advantage of:}

- Convenient online submission

- Thorough peer review

- No space constraints or color figure charges

- Immediate publication on acceptance

- Inclusion in PubMed, CAS, Scopus and Google Scholar

- Research which is freely available for redistribution 